\section{Frequência de problemas vocais autorreferidos e fatores ocupacionais associados em professores da educação básica de Londrina, Paraná, Brasil}

\author{
Frequency of self-reported vocal problems \\ and associated occupational factors in primary \\ schoolteachers in Londrina, Paraná State, Brazil \\ Frecuencia de problemas vocales autorreportados \\ y factores ocupacionales asociados en maestros de \\ la enseñanza básica en Londrina, Paraná, Brasil
}

Michelle Moreira Abujamra Fillis 1,2 Selma Maffei de Andrade 2 Alberto Durán González 2 Francine Nesello Melanda 2 Arthur Eumann Mesas 2

\begin{abstract}
Resumo
O objetivo deste trabalho foi estimar a prevalência da percepção de problemas vocais frequentes em professores da educação básica e investigar os fatores ocupacionais associados. Estudo transversal, realizado mediante entrevistas de 967 professores atuantes em 20 escolas estaduais de Londrina, Paraná, Brasil. A percepção de problemas vocais frequentes foi de 25,7\%. Análises ajustadas mostraram associação desses problemas com características do vínculo de trabalho ( $\geq 40$ horas/semana, percepção ruim da remuneração e dos benefícios de saúde), características do ambiente de trabalho (quantidade de alunos por sala, exposição a pó de giz e microorganismos), aspectos psicológicos (menor realização profissional, baixa oportunidade de expressar opiniões, pior relacionamento com superiores e equilíbrio entre vida profissional e pessoal) e situações de violência (insultos e assédio moral). A percepção de transtornos vocais frequentes afeta um em cada quatro professores da educação básica e está associada a diversas características da atividade docente, tanto estruturais como referentes ao processo de trabalho.
\end{abstract}

Docentes; Distúrbios da Voz; Ensino Fundamental e Médio; Saúde do Trabalhador

\footnotetext{
1 Universidade do Norte do Paraná, Londrina, Brasil.

2 Universidade Estadual de

Londrina, Londrina, Brasil.

\author{
Correspondência \\ M. M. A. Fillis \\ Rua Weslley Cesar Vanzo 189 \\ Londrina, $P R$ \\ 86050-500, Brasil.
}

micmoreira@gmail.com
} 


\section{Introdução}

A escola constitui um ambiente importante na configuração da realidade de vida do professor e dos aspectos relacionados às condições e organização do trabalho docente, os quais repercutem sobre o processo saúde-doença 1 .

A categoria profissional dos professores é uma das mais acometidas por alterações vocais, apresentando como causas tanto as condições nas quais o trabalho é desenvolvido quanto à forma como ele é organizado e estabelecido no processo educacional. As condições de trabalho remetem à estrutura física na qual se exerce a atividade docente, ou seja, às condições materiais e ambientais em que o trabalho é realizado. Já as características inerentes à organização do trabalho referem-se à forma como as atividades estão discriminadas, como os tempos estão divididos, a distribuição das tarefas e competências, as relações de hierarquia que refletem relações de poder, dentre outros 2 .

Na profissão docente, a voz é fator relevante para o desempenho profissional e para a atuação do professor em sala de aula, especialmente como componente constitutivo da identidade do professor como trabalhador, do impacto do tom da voz do docente sobre o discente e do componente do processo ensino-aprendizagem ${ }^{3}$.

A etiologia da disfonia é multidimensional. Segundo Ferreira et al. 4, os riscos ocupacionais que trazem prejuízos à voz e à saúde dos docentes podem estar relacionados ao ambiente de trabalho (ruído no ambiente escolar, choque térmico, ventilação inadequada do ambiente, exposição a produtos irritativos das vias aéreas superiores e presença de poeira no local de trabalho) e à organização do trabalho (jornada de trabalho prolongada, acúmulo de atividades ou de funções, demanda vocal excessiva, ausência de pausas durante a jornada, falta de autonomia). Outros aspectos a serem considerados, além do risco ocupacional, são os biológicos como o envelhecimento, alergias, infecções das vias aéreas superiores, refluxo laringofaríngeo, influências hormonais, além do uso de medicações, etilismo, tabagismo e falta de hidratação 4 .

Estudos que investigaram os distúrbios da voz em professores relataram prevalência de $4,4 \%$ a $90 \% 5$. Especificamente em estudos brasileiros, a prevalência de alteração vocal variou de $10,6 \%$ a $87 \%$ em professores de redes municipais e estaduais, do ensino infantil e fundamental 2,6,7. Ainda não está claro na literatura quais são as condições de trabalho e estilo de vida associados com a percepção de problemas com a voz nessa categoria profissional 5 . Gianini et al. 8, em um estudo com professoras da rede municipal de São Paulo, observaram associação entre a presença de distúrbios da voz (identificados mediante avaliação vocal e laringoscópica) e estresse no trabalho ou, mais especificamente, com a condição representada pela percepção de alta demanda e baixo controle no trabalho. Outros estudos apontam, ainda, que certas condições de trabalho e fatores do ambiente escolar, tais como questões emocionais, esgotamento mental e estresse, doenças do aparelho respiratório e vias aéreas superiores podem contribuir para o desenvolvimento de distúrbio da voz ${ }^{9,10}$.

Para examinar a relação entre aspectos ocupacionais e alterações vocais é importante considerar o possível efeito confundidor de variáveis como gênero, idade, etilismo, tabagismo e alterações respiratórias nestas relações, uma vez que tais variáveis podem associar-se tanto com alterações vocais como com certas variáveis relacionadas ao trabalho. Por um lado, estudos indicaram haver relação entre alterações vocais e gênero 11 , idade 12 , etilismo 13 , alterações respiratórias e tabagismo 4 . Por outro, as condições de trabalho estão associadas ao gênero ${ }^{14}$, idade 15 , problemas respiratórios 15 , etilismo ${ }^{16}$ e tabagismo 16 .

Diante do exposto, este trabalho teve como objetivo identificar a prevalência da percepção de problemas vocais frequentes de professores da educação básica, investigar sua associação com fatores ocupacionais subjetivos e objetivos, e examinar se tais associações são independentes de seus principais fatores de confusão.

\section{Métodos}

Trata-se de um estudo transversal, parte de um projeto maior intitulado Pró-Mestre - Saúde, Estilo de Vida e Trabalho de Professores da Rede Pública do Paraná, conduzido no Município de Londrina, Paraná, Brasil.

A população do estudo foi composta por professores do ensino fundamental ou médio da rede estadual de ensino de Londrina. Inicialmente, a direção do Núcleo Regional da Secretaria de Educação do Paraná foi contatada para a apresentação do projeto e houve anuência ao pedido de contato com as escolas. Também foi solicitada uma lista das escolas em ordem decrescente de número de professores, que variavam de 133 a 9 , todas de Ensino Fundamental (ciclo II - do sexto ao nono ano) e Médio. Foram selecionadas as 20 escolas com o maior número de professores. O acesso às escolas foi realizado por meio de uma reunião com os diretores, na qual foram expostos os objetivos e a metodologia do projeto. Em seguida, foi solicitada uma lista com o nome de todos os professores que naquele momento atua- 
vam na escola, independentemente dos critérios de inclusão e exclusão utilizados posteriormente.

O convite aos professores foi feito em um momento de sensibilização e apresentação do projeto, no intervalo entre as aulas e nos três turnos (manhã, tarde e noite), com exposição de um pôster e distribuição de panfletos contendo informações sobre a pesquisa. Em seguida, aqueles professores que cumpriam os critérios de inclusão foram contatados e convidados individualmente por integrantes da equipe.

Foram considerados como critérios de inclusão: atuar em sala de aula no ensino regular Fundamental ou Médio e ser responsável por uma disciplina. Considerou-se como perdas as situações nas quais os professores não aceitaram participar da pesquisa e cujo contato não foi possível após cinco tentativas, em diferentes dias e horários. Um período de 21 dias foi estabelecido para a coleta de dados em cada escola. Na tentativa de localizar professores que estavam em licença durante esse período e não puderam ser entrevistados, retornou-se às escolas após 15 e 30 dias, contados a partir do término do período de coleta dos dados. Se ainda assim esses docentes não tivessem retornado às suas atividades, também foram considerados perdas. Excluíram-se os professores com tempo de profissão menor que um ano, no sentido de padronizar o tempo de exposição à atividade docente em, no mínimo, 1 ano.

O instrumento para a coleta de dados foi elaborado com base na literatura e previamente testado em um estudo piloto com 82 professores de três escolas estaduais do Município de Cambé (Paraná). Após ajustes, a versão definitiva foi constituída por duas partes: um formulário para entrevista, cujas respostas eram anotadas pelo entrevistador, com questões majoritariamente objetivas referentes à percepção de frequência de problemas vocais, condições de trabalho, estilo de vida, saúde e violência escolar, entre outras variáveis; um questionário composto por questões fechadas sobre variáveis sociais, demográficas, entre outras, foi respondido em papel diretamente pelo professor após a entrevista, que foi previamente agendada e realizada preferencialmente durante a hora-atividade, em locais reservados, nas instalações da escola de atuação do professor. A coleta de dados ocorreu entre agosto de 2012 e junho de 2013, e foi realizada por alunos da graduação de enfermagem e medicina, e pós-graduação em saúde coletiva da Universidade Estadual de Londrina (UEL).

As variáveis consideradas neste estudo foram as características sociodemográficas (gênero e idade), do vínculo de trabalho (tempo de profissão, número de locais onde trabalha, carga horária de trabalho como professor, nível de ensino que leciona, remuneração em relação ao trabalho realizado, benefícios de saúde oferecidos e tipo de contrato), características do ambiente de trabalho (quantidade de alunos/sala de aula; exposição a ruídos dentro da sala, na escola e fora da escola; exposição a pó de giz e a poeiras; condições de higiene no seu local de trabalho; exposições a vírus, bactérias, fungos e parasitas), aspectos psicológicos do trabalho (realização profissional; relacionamento com superiores, alunos e professores; oportunidade de expressar suas opiniões no trabalho e equilíbrio entre a vida pessoal e profissional), aspectos da organização do trabalho (ritmo e intensidade do seu trabalho; autonomia para desempenhar tarefas; número e responsabilidade que elas exigem e tempo disponível para as tarefas realizadas no seu trabalho; atenção e responsabilidade que elas exigem; e tempo disponível para o preparo das atividades) e violência escolar contra professores (insultos, assédio moral e violência física).

Para a análise de fatores associados, a variável dependente "percepção de problemas vocais (PPV)" foi agrupada em frequente (sempre e frequentemente) e não frequente (às vezes, raramente ou nunca).

Os dados foram duplamente digitados em banco criado no programa Epi Info, versão 3.5.4 (Centers for Disease Control and Prevention, Atlanta, Estados Unidos), e analisados usandose o programa SPSS, versão 19.0 (IBM Corp., Armonk, Estados Unidos). A análise descritiva foi realizada por meio de frequências absolutas e relativas, medidas de tendência central e de dispersão. Para a análise bivariada utilizou-se a razão de prevalência (RP) como medida de associação, e foi adotado o nível de significância de 5\% (teste qui-quadrado de Wald com apresentação do valor de p e do intervalo de $95 \%$ de confiança - IC95\%). Para as análises ajustadas construíramse modelos de regressão de Poisson, nos quais a variável dependente era sempre a PPV frequente, e apenas uma condição de trabalho era incluída como variável independente, além das variáveis de ajuste gênero e idade, uso de tabaco, álcool e alterações respiratórias. Para todas as variáveis foi estabelelecida uma categoria de referência $(\mathrm{RP}=1)$, considerada a de menor risco para a ocorrência do desfecho. Também foram testadas as interações entre todos os fatores ocupacionais e o gênero e a idade na relação com a PPV frequente por meio de testes de razão de verossimilhança, que comparam modelos com e sem os termos de interação.

O projeto foi aprovado pelo Comitê de Ética em Pesquisa da UEL (CAAE no 01817412.9.0000. 5231) e cada entrevista foi conduzida somente 
após o esclarecimento dos objetivos da pesquisa e consentimento do participante, mediante assinatura do Termo de Consentimento Livre e Esclarecido.

\section{Resultados}

Dos 1.505 professores que tinham vínculo profissional com as escolas, 379 foram excluídos por integrarem apenas o quadro de docente do ensino técnico ou do ensino complementar ( $\mathrm{n}=$ 254), ou por estarem afastados ou readaptados de função $(n=125)$, restando 1.126 docentes elegíveis para a pesquisa. Entre os 1.126 professores que atuavam em sala de aula ao menos um período por semana, 63 (5,6\%) recusaram-se a participar, 65 (5,7\%) encontravam-se em licença e não retornaram após 51 dias do início da coleta de dados em sua escola, e 20 (1,8\%) não foram encontrados após a quinta tentativa, totalizando 148 perdas $(13,1 \%)$. Considerando o período de exposição às cargas ocupacionais, 9 professores foram excluídos destas análises por apresentarem tempo de profissão menor que 12 meses e 2 por ausência de informação sobre voz. Assim, a população final foi de 967 professores. Na população estudada, $666(68,9 \%)$ docentes eram do gênero feminino, 475 (49,1\%) casados, 716 (74\%) de raça/cor branca e 695 (71,9\%) com grau de instrução especialização. A média da idade foi de 41,6 \pm 9,9 anos (mediana de 42 anos, mínimo de 23 e máximo de 68). A média do tempo de profissão como professor foi de 13,0 \pm 9,0 anos (mediana de 12 anos, mínimo de 1 e máximo de 45 anos). Com referência ao tipo de vínculo de trabalho, 667 (69\%) dos professores tinham ao menos 1 vínculo como estatutário e os $31 \%$ restantes atuavam com contrato por tempo determinado (PSS). A carga horária como professor (horas de atuação como professor em todos os vínculos) predominante foi de 40 horas por semana (26,7\%). Quanto ao período de trabalho, destacou-se a maior frequência da atuação profissional em dois turnos (64,2\%), e 16,7\% atuavam nos três turnos.

Dos docentes entrevistados, 25,7\% ( $n=249$ ) referiram apresentar problemas com a voz sempre ou frequentemente, e $74,3 \%(n=718)$ referiram estes problemas às vezes, raramente ou nunca. A maior prevalência de PPV frequente foi observada para o gênero feminino $(\mathrm{p}<0,001)$, e não houve diferença estatisticamente significativa segundo a idade.

Na Tabela 1, observa-se maior prevalência de problemas com a voz em professores que têm uma carga horária igual ou maior que 40 horas semanais e que consideram a remuneração e os benefícios de saúde oferecidos como regulares ou ruins. Características específicas do ambiente também foram associadas com maior frequência de problemas com a voz, como a quantidade de alunos em sala de aula, as condições de higiene da escola e a exposição ao pó de giz e a microorganismos (Tabela 2).

A percepção negativa quanto à realização profissional e ao equilíbrio entre a vida pessoal e profissional, assim como o pior relacionamento com os superiores (diretores) e dificuldade para expressar suas opiniões no trabalho também estiveram associados com a maior prevalência de PPV frequente (Tabela 3).

Finalmente, na Tabela 4, observam-se associações significativas entre a PPV frequente e o número de tarefas realizadas no trabalho e situações de violência escolar sofridas pelo professor na escola, como insultos e assédio moral.

Todas as interações testadas entre os aspectos ocupacionais estudados e as variáveis gênero e idade não foram estatisticamente significativas ( $\mathrm{p}>0,05)$.

\section{Discussão}

Este estudo identificou que um de cada quatro professores da Educação Básica muito frequentemente apresenta problemas relacionados com a voz durante o trabalho. Além disso, fatores ocupacionais relacionados com a organização e condições do trabalho e com a percepção do professor quanto à sua exposição ao ambiente $\mathrm{e}$ às cargas de trabalho estiveram associados com o aumento da percepção de problemas vocais frequentes. O conceito de alteração vocal assumido neste trabalho não tem a pretensão diagnóstica de uma disfonia, mas sim como indicativo de um possível distúrbio de voz relacionado ao trabalho.

Estudos que tomam como critério a autorreferência de alterações vocais observaram prevalências muito diversas, variando entre $11 \% \mathrm{e}$ $80,7 \%$ 2,5,6,7. Essa variação é resultante, sobretudo, dos métodos, instrumentos e definições de disfonia empregados. A comparação entre estudos sobre a prevalência de alteração vocal em professores é difícil devido aos diferentes métodos de avaliação. Em muitos estudos epidemiológicos a alteração vocal é definida pela referência de sintomas vocais $2,5,6,7$, por outro lado, outras vezes a prevalência desta alteração pode ser subestimada se a avaliação da voz se restringir ao exame físico da laringe 17 .

Lima-Silva et al. 18 confirmaram maior autorreferência de distúrbio da voz por parte dos professores participantes do que o constatado pela avaliação perceptiva da voz e de pregas vocais, 
Razão de prevalência (RP) e intervalo de 95\% de confiança (IC95\%) da percepção de problemas vocais frequentes, com ajuste de gênero, idade, uso de tabaco, álcool e alterações respiratórias, em professores segundo características do vínculo de trabalho. Londrina, Paraná, Brasil, 2012-2013.

\begin{tabular}{|c|c|c|c|c|c|c|}
\hline \multirow[t]{2}{*}{ Características do trabalho } & \multicolumn{6}{|c|}{ Percepção de problemas vocais frequentes } \\
\hline & Total & $\mathbf{n}$ & $\%$ & RP & IC95\% & Valor de $p$ \\
\hline \multicolumn{7}{|l|}{ Tempo de profissão (anos) } \\
\hline$<12$ & 462 & 107 & 23,2 & 1,00 & & \\
\hline$\geq 12$ & 505 & 142 & 28,1 & 1,16 & $0,87-1,57$ & 0,314 \\
\hline \multicolumn{7}{|l|}{ Número de locais onde trabalha } \\
\hline 1 & 262 & 78 & 29,8 & 1,00 & & \\
\hline Mais que 1 & 705 & 171 & 24,3 & 0,92 & $0,73-1,15$ & 0,436 \\
\hline \multicolumn{7}{|c|}{ Carga horária de trabalho como professor (horas) } \\
\hline$<40$ & 506 & 109 & 21,5 & 1,00 & & \\
\hline$\geq 40$ & 461 & 140 & 30,4 & 1,46 & $1,18-1,80$ & 0,001 \\
\hline \multicolumn{7}{|c|}{ Quantidade de turnos em que trabalha } \\
\hline 1 & 184 & 46 & 25,0 & 1,00 & & \\
\hline 2 ou mais & 783 & 203 & 25,7 & 1,05 & $0,80-1,37$ & 0,728 \\
\hline \multicolumn{7}{|l|}{ Nível de ensino em que leciona } \\
\hline Outros níveis de ensino & 252 & 50 & 19,8 & 1,00 & & \\
\hline Ensino Fundamental & 715 & 199 & 27,8 & 1,23 & $0,93-1,61$ & 0,146 \\
\hline \multicolumn{7}{|c|}{ Remuneração em relação ao trabalho realizado } \\
\hline Bom/Excelente & 358 & 72 & 20,1 & 1,00 & & \\
\hline Ruim/Regular & 608 & 177 & 29,1 & 1,38 & $1,09-1,75$ & 0,007 \\
\hline \multicolumn{7}{|l|}{ Benefícios de saúde oferecidos } \\
\hline Bom/Excelente & 59 & 7 & 11,9 & 1,00 & & \\
\hline Ruim/Regular & 868 & 230 & 26,5 & 2,02 & $1,02-3,99$ & 0,043 \\
\hline \multicolumn{7}{|l|}{ Tipo de contrato } \\
\hline Estatutário & 667 & 182 & 27,3 & 1,00 & & \\
\hline Não estatutário & 300 & 67 & 22,3 & 0,79 & $0,62-1,02$ & 0,071 \\
\hline
\end{tabular}

provavelmente pela presença de sintomas que ainda não se manifestaram clinicamente como alteração da voz ou da laringe. A queixa autorreferida do distúrbio da voz evidencia a exposição a condições adversas de trabalho, e a percepção do professor é um instrumento fundamental para o diagnóstico precoce da disfunção vocal. Além disso, deve-se ressaltar a importância de ações de promoção de bem-estar vocal que disseminem orientações aos profissionais da voz no tratamento imediato ao aparecimento dos sintomas vocais, como forma de prevenir futuras alterações 4,18,19.

A população deste estudo é composta predominantemente por mulheres, casadas, com formação superior e que ensinam no nível fundamental. A maior prevalência de PPV frequente observada nesse gênero poderia ser parcialmente explicada por fatores individuais, emocionais e sociais. A susceptibilidade biológica (dimen- são reduzida da laringe e influência hormonal) e o papel social que exercem, com sobrecarga de atividades profissionais, domésticas e pessoais favorecem a maior vulnerabilidade de mulheres no exercício da docência 11 . O resultado do acúmulo de papéis produz a chamada dupla jornada: a da atividade profissional e a das atividades domésticas, como mãe e dona de casa. Essa dupla jornada significa uma intensa carga horária de trabalho para a mulher, contribuindo para o desenvolvimento de doenças, principalmente aquelas relacionadas ao estresse 20 .

Contrariamente ao esperado, não observamos relação entre a PPV frequente e a idade ou tempo de profissão. Acredita-se que isso se deva ao fato dos professores com problemas importantes de voz serem "readaptados" (afastados do magistério e designados para outras funções no setor da educação) ou até mesmo abandonarem a profissão, em casos mais extremos. Urrutiko- 
Razão de prevalência (RP) e intervalo de 95\% de confiança (IC95\%) da percepção de problemas vocais frequentes, com ajuste de gênero, idade, uso de tabaco e álcool e alterações respiratórias em professores segundo características do ambiente de trabalho. Londrina, Paraná, Brasil, 2012-2013.

\begin{tabular}{|c|c|c|c|c|c|c|}
\hline \multirow[t]{2}{*}{ Características do trabalho } & \multicolumn{6}{|c|}{ Percepção de problemas vocais frequentes } \\
\hline & Total & $\mathrm{n}$ & $\%$ & RP & IC95\% & Valor de $\mathrm{p}$ \\
\hline \multicolumn{7}{|c|}{ Quantidade de alunos/sala de aula } \\
\hline Bom/Excelente & 303 & 57 & 18,8 & 1,00 & & \\
\hline Ruim/Regular & 664 & 192 & 28,9 & 1,44 & $1,11-1,87$ & 0,006 \\
\hline \multicolumn{7}{|c|}{ Exposição a ruídos dentro da sala } \\
\hline Não afeta & 49 & 6 & 12,2 & 1,00 & & \\
\hline Afeta & 918 & 243 & 26,5 & 1,95 & $0,92-4,16$ & 0,083 \\
\hline \multicolumn{7}{|l|}{ Exposições a ruídos na escola } \\
\hline Não afeta & 147 & 35 & 23,8 & 1,00 & & \\
\hline Afeta & 820 & 214 & 26,1 & 1,05 & $0,77-1,42$ & 0,772 \\
\hline \multicolumn{7}{|c|}{ Exposição a ruídos que vêm de fora da escola } \\
\hline Não afeta & 418 & 103 & 24,6 & 1,00 & & \\
\hline Afeta & 549 & 146 & 26,6 & 1,05 & $0,84-1,30$ & 0,691 \\
\hline \multicolumn{7}{|l|}{ Exposição a pó de giz } \\
\hline Não afeta & 210 & 35 & 16,7 & 1,00 & & \\
\hline Afeta & 756 & 214 & 28,3 & 1,48 & $1,07-2,04$ & 0,017 \\
\hline \multicolumn{7}{|l|}{ Exposição a poeiras } \\
\hline Não afeta & 232 & 43 & 18,5 & 1,00 & & \\
\hline Afeta & 734 & 206 & 28,1 & 1,31 & $0,98-1,76$ & 0,073 \\
\hline \multicolumn{7}{|c|}{ Condições de higiene do seu local de trabalho } \\
\hline Não afeta & 388 & 77 & 19,8 & 1,00 & & \\
\hline Afeta & 579 & 172 & 29,7 & 1,43 & $1,13-1,80$ & 0,003 \\
\hline \multicolumn{7}{|c|}{ Exposições a vírus, bactérias, fungos e parasitas } \\
\hline Não afeta & 338 & 65 & 19,2 & 1,00 & & \\
\hline Afeta & 629 & 184 & 29,3 & 1,41 & $1,10-1,81$ & 0,006 \\
\hline
\end{tabular}

etxea et al. 12 acreditam que, com o passar dos anos, o professor realize um autocontrole involuntário, melhorando sua técnica vocal de maneira espontânea. Isso justificaria a diminuição de incidência de nódulos vocais à proporção que aumenta o número de anos de atividade profissional. Quanto ao tempo médio de atuação dos docentes, não houve associação estatisticamente significativa entre a prevalência de PPV frequente e atuar por 7 anos ou mais, em comparação com o tempo de 1 a menos de 7 anos. Outros estudos, assim como este, não confirmam a associação de tempo de magistério e alterações vocais 21,22.

A carga horária semanal predominante foi de 40 horas e estava significativamente associada com a percepção de problemas vocais frequentes, assim, é possível sugerir que quanto maior a carga horária, maior o uso da voz e maior o seu desgaste, podendo ocasionar fadiga vocal. Essa associação é concordante com o estudo de Fuess \& Lorenz 22 e com o de Provenzano \& Sampaio 23.
Nesse último, as autoras apontaram que $78 \%$ dos docentes afastados de sala de aula no Estado do Rio de Janeiro tinham carga horária igual ou maior que 40 horas semanais.

Um estudo sobre o nível de ensino sugere que os distúrbios da voz são mais prevalentes entre os professores que lecionam para alunos mais jovens 23 . Foi sugerido que a carga vocal entre docentes de estudantes pré-escolares e de níveis primários é maior do que a de professores do Ensino Médio, porque, além de ter de competir com o ruído produzido pelas crianças durante períodos de tempo mais longos, as pausas para descanso são mais escassas, uma vez que, geralmente, todas as matérias são ministradas pelos mesmos docentes 24,25. No entanto, neste estudo os professores das séries fundamentais não apresentam maior prevalência da PPV frequente do que aqueles que atuam também ou exclusivamente em séries mais avançadas, como no nível médio e/ou técnico. 
Razão de prevalência (RP) e intervalo de 95\% de confiança (IC95\%) da percepção de problemas vocais frequentes, com ajuste de gênero, idade, uso de tabaco e álcool e alterações respiratórias em professores segundo aspectos psicológicos do trabalho. Londrina, Paraná, Brasil, 2012-2013.

\begin{tabular}{|c|c|c|c|c|c|c|}
\hline \multirow[t]{2}{*}{ Características do trabalho } & \multicolumn{6}{|c|}{ Percepção de problemas vocais frequentes } \\
\hline & Total & $\mathbf{n}$ & $\%$ & $\mathrm{RP}$ & IC95\% & Valor de $\mathrm{p}$ \\
\hline \multicolumn{7}{|l|}{ Realização profissional } \\
\hline $\operatorname{Sim}$ & 431 & 90 & 20,9 & 1,00 & & \\
\hline Parcialmente/Não & 536 & 159 & 29,7 & 1,38 & $1,10-1,73$ & 0,005 \\
\hline \multicolumn{7}{|l|}{ Relacionamento com superiores } \\
\hline Bom/Excelente & 892 & 220 & 24,7 & 1,00 & & \\
\hline Ruim/Regular & 75 & 29 & 38,7 & 1,60 & $1,19-2,14$ & 0,002 \\
\hline \multicolumn{7}{|l|}{ Relacionamento com alunos } \\
\hline Bom/Excelente & 866 & 226 & 26,1 & 1,00 & & \\
\hline Ruim/Regular & 101 & 23 & 22,8 & 0,90 & $0,63-1,30$ & 0,583 \\
\hline \multicolumn{7}{|c|}{ Relacionamento com outros professores } \\
\hline Bom/Excelente & 930 & 242 & 26,0 & 1,00 & & \\
\hline Ruim/Regular & 37 & 7 & 18,9 & 0,78 & $0,39-1,564$ & 0,482 \\
\hline \multicolumn{7}{|c|}{ Oportunidade de expressar suas opiniões no trabalho } \\
\hline Bom/Excelente & 782 & 183 & 23,4 & 1,00 & & \\
\hline Ruim/Regular & 179 & 65 & 36,3 & 1,53 & $1,23-1,92$ & $<0,001$ \\
\hline \multicolumn{7}{|c|}{ Equilíbrio entre sua vida profissional e pessoal } \\
\hline Bom/Excelente & 695 & 162 & 23,3 & 1,00 & & \\
\hline Ruim/Regular & 272 & 87 & 32,0 & 1,28 & $1,03-1,60$ & 0,029 \\
\hline
\end{tabular}

A prevalência da percepção de problemas vocais frequentes foi associada à pior avaliação da remuneração pelo trabalho realizado. A renda é considerada um dos fatores determinantes do processo saúde-doença: pessoas com renda mais baixa adoecem com maior frequência, têm menos resistência e estão expostas a vários fatores de riscos à saúde 26 .

O trabalho docente é uma atividade centrada nas relações interpessoais e nas dinâmicas relacionais estabelecidas no ambiente escolar, que são determinantes do sucesso do ensino e da qualidade de vida do professor 27 . Na análise das relações interpessoais no ambiente de trabalho, envolvendo a direção da escola e os próprios alunos, a PPV frequente esteve associada com a pior avaliação da relação com os superiores. Jardim et al. 28 mencionam que diante dos sintomas vocais o professor começa a ter dificuldades de manifestações e relações interpessoais com os demais profissionais envolvidos na escola.

A PPV frequente esteve associada com a pior avaliação da quantidade de alunos por sala de aula. Esse resultado corrobora outro estudo comparativo com uma mesma população de professores em períodos diferentes, no qual se consta- tou que um dos importantes fatores de risco para alteração vocal foi o aumento do número de alunos em sala no decorrer do tempo ${ }^{29}$. Nesse sentido, a competição da voz com o ruído ambiental produzido por mais alunos pode favorecer a presença de esforço intenso para se fazer ouvir 30 . No entanto, é possível que haja outros aspectos envolvidos e não considerados neste estudo, uma vez que, contrariando a hipótese inicial, não se verificou associação entre maior frequência de problemas vocais e exposição a ruídos dentro da sala de aula.

A análise atual evidenciou associação significativa entre a percepção de problemas vocais frequentes e o incômodo causado pela presença de pó de giz. Tal associação pode ser explicada pela irritação da mucosa nasal e laríngea devido à inalação da poeira de giz 31 . Ainda quanto ao ambiente de trabalho, verificou-se que $28,1 \%$ dos professores relataram que são afetados pela exposição a poeiras, $29,8 \%$ pelas condições de higiene do seu local de trabalho e também pela exposição a vírus, bactérias, fungos e parasitas (29,3\%). Sabe-se que a exposição da laringe a irritantes de mucosa, por exemplo, poeira ou mofo, influencia negativamente o mecanismo vocal 32,33 . 
Razão de prevalência (RP) e intervalo de 95\% de confiança (IC95\%) da percepção de problemas vocais frequentes, com ajuste de gênero, idade, uso de álcool e tabaco em professores segundo aspectos organizacionais e violência sofrida pelo professor no local de trabalho, Londrina, Paraná, Brasil, 2012-2013.

\begin{tabular}{|c|c|c|c|c|c|c|}
\hline \multirow[t]{2}{*}{ Características do trabalho } & \multicolumn{6}{|c|}{ Percepção de problemas vocais frequentes } \\
\hline & Total & n & $\%$ & RP & IC95\% & Valor de $\mathrm{p}$ \\
\hline \multicolumn{7}{|c|}{ Ritmo e intensidade do seu trabalho } \\
\hline Não afeta & 116 & 22 & 19,0 & 1,00 & & \\
\hline Afeta & 851 & 227 & 26,7 & 1,38 & $0,94-2,03$ & 0,105 \\
\hline \multicolumn{7}{|c|}{ Autonomia para desempenhar tarefas } \\
\hline Não afeta & 477 & 110 & 23,1 & 1,00 & & \\
\hline Afeta & 490 & 139 & 28,4 & 1,18 & $0,96-1,46$ & 0,124 \\
\hline \multirow{2}{*}{\multicolumn{7}{|c|}{$\begin{array}{l}\text { Número de tarefas realizadas no seu trabalho e a atenção e } \\
\text { responsabilidade que elas exigem }\end{array}$}} \\
\hline & & & & & & \\
\hline Não afeta & 146 & 25 & 17,1 & 1,00 & & \\
\hline Afeta & 820 & 224 & 27,3 & 1,59 & $1,10-2,29$ & 0,014 \\
\hline \multicolumn{7}{|c|}{ Tempo disponível para preparo de atividades } \\
\hline Não afeta & 109 & 23 & 21,1 & 1,00 & & \\
\hline Afeta & 858 & 226 & 26,3 & 1,16 & $0,80-1,68$ & 0,431 \\
\hline \multicolumn{7}{|l|}{ Insultos } \\
\hline Não & 443 & 95 & 21,4 & 1,00 & & \\
\hline Sim & 524 & 154 & 29,4 & 1,29 & $1,04-1,61$ & 0,023 \\
\hline \multicolumn{7}{|l|}{ Assédio moral } \\
\hline Não & 793 & 191 & 24,1 & 1,00 & & \\
\hline Sim & 174 & 58 & 33,3 & 1,36 & $1,07-1,73$ & 0,011 \\
\hline \multicolumn{7}{|l|}{ Violência física } \\
\hline Não & 888 & 222 & 25,0 & 1,00 & & \\
\hline Sim & 79 & 27 & 34,2 & 1,25 & $0,92-1,70$ & 0,163 \\
\hline
\end{tabular}

A percepção de distúrbios vocais frequentes também esteve associada com o relato de situações de violência sofridas pelo professor no ambiente escolar, como insultos e assédio moral. A autorreferência à presença do distúrbio da voz evidencia a exposição a condições adversas de trabalho e o docente dificilmente consegue reverter seu quadro de adoecimento. Instrumento fundamental na mediação, a voz do professor é, sem dúvida, um potente recurso na relação com os alunos. Num contexto de indisciplina, é comum recorrer ao uso de uma expressão vocal que imponha um fazer imperativo como gritar para pedir atenção, ou falar sem parar para garantir seu turno 34 .

Em síntese, este estudo identificou elevada prevalência de percepção de transtornos vocais frequentes em professores da Educação Básica, e destaca um conjunto de condições de trabalho associadas a estes problemas. Além das características do vínculo de trabalho, como carga horária e quantidade de alunos por sala de aula, certas condições estruturais da escola, a exposição a cargas físicas e psíquicas e sofrer situações de violência escolar são aspectos que merecem destaque na prevenção e no tratamento de problemas vocais entre os docentes. Embora algumas dessas associações tenham sido constatadas em estudos anteriores, a disfonia ainda não é formalmente considerada como uma doença ocupacional. Assim, estudos prospectivos são necessários para aprofundar o conhecimento sobre os determinantes das alterações da voz em professores, sobretudo os relacionados com a atividade docente em sala de aula. 


\section{Colaboradores}

M. M. A. Fillis contribuiu com a concepção e delineamento, coleta de dados, análise estatística, preparação do manuscrito, redação, revisão crítica e aprovação final. S. M. Andrade contribuiu com a concepção e delineamento, preparação do manuscrito, redação e revisão crítica. A. D. González contribuiu com a concepção e delineamento, preparação e redação do manuscrito. F. N. Melanda contribuiu com a concepção e delineamento, aquisição e interpretação dos dados, análise estatística e revisão do artigo. A. E. Mesas contribuiu com a concepção e delineamento, preparação do manuscrito, redação, revisão crítica e aprovação final.

\section{Referências}

1. Penteado RZ, Pereira IMTB. Qualidade de vida e saúde vocal de professores. Rev Saúde Pública 2007; 41:236-43.

2. Brasil RN. Fatores associados a alterações vocais em professores de Salvador [Dissertação de Mestrado]. Salvador: Programa de Pós-graduação em Saúde, Ambiente e Trabalho, Faculdade de Medicina da Bahia, Universidade Federal da Bahia; 2010.

3. Ricarte A, Bommarito S, Chiari B. Impacto vocal de professores. Rev CEFAC 2011; 13:719-27.

4. Ferreira LP, Alves IAV, Esteves AAO, Biserra MP. Voz do professor: fatores predisponentes para o bemestar vocal. Distúrb Comun 2012; 24:379-87.

5. Cantor Cutiva LC, Vogel I, Burdorf A. Voice disorders in teachers and their associations with workrelated factors: a systematic review. J Commun Disord 2013; 46:143-55.

6. Alves LP, Araújo LTR, Xavier Neto JA. Prevalência de queixas vocais e estudo de fatores associados em uma amostra de professores de ensino fundamental em Maceió, Alagoas, Brasil. Rev Bras Saúde Ocup 2010; 35:168-75.

7. Musial PL, Dassie-Leite AP, Zaboroski AP, Casagrande RC. Interferência dos sintomas vocais na atuação profissional de professores. Distúrb Comun 2011; 23:335-41.

8. Giannini SPP, Latorre MRD, Ferreira LP. Distúrbio de voz e estresse no trabalho docente: um estudo caso-controle. Cad Saúde Pública 2012; 28: 2115-24.

9. Gonçalves CGO, Penteado RZ, Silvério KCA. Fonoaudiologia e saúde do trabalhador: a questão da saúde vocal do professor. Saúde Rev 2005; 7:45-51.

10. Dragone MLS, Ferreira LP, Giannini SPP, Simões-Zenari M, Vieira VP, Behlau M. Voz do professor: uma revisão de 15 anos de contribuição fonoaudiológica. Rev Soc Bras Fonoaudiol 2010; 15: 289-96.

11. Calas M, Verhulst J, Lecoq M, Dalleas B, Seilhean M. Vocal pathology of teachers. Rev Laryngol Otol Rhinol (Bord) 1988; 110:397-406.

\section{Agradecimentos}

Este estudo foi parcialmente financiado com recursos obtidos junto à Fundação Araucária do Paraná (processo no 168/2014). Os autores agradecem aos professores da rede pública estadual de Londrina pela imprescindível colaboração.
12. Urrutikoetxea A, Ispizua A, Matellanes F. Vocal pa thology in teachers: a videolaryngostroboscopic study in 1046 teachers. Rev Laryngol Otol Rhinol (Bord) 1994; 116:255-62.

13. Lee SY, Lao XQ, Yu IT. A cross-sectional survey of voice disorders among primary school teachers in Hong Kong. J Occup Health 2010; 52:344-52.

14. Araújo TM, Godinho TM, Reis EJFB, Almeida MMG. Diferenciais de gênero no trabalho docente e repercussões sobre a saúde. Ciênc Saúde Coletiva $2006 ; 11: 1117-29$.

15. Gasparini SM, Barreto SM, Assunção AA. O professor, as condições de trabalho e os efeitos sobre sua saúde. Educação e Pesquisa 2005; 31:189-99.

16. Martinez MC, Latorre M, Fischer FM. Capacidade para o trabalho: revisão de literatura. Ciênc Saúde Coletiva 2010; 15:1553-61.

17. Mathieson L, Hirani S, Epstein R, Baken R, Wood G, Rubin J. Laryngeal manual therapy: a preliminary study to examine its treatment effects in the management of muscle tension dysphonia. J Voice 2009; 23:353-66.

18. Lima-Silva MFB, Ferreira LP, Oliveira IB, Silva MAA, Ghirardi ACAM. Distúrbio de voz em professores: autorreferência, avaliação perceptiva da voz e das pregas vocais. Rev Soc Bras Fonoaudiol 2012; 17:391-7.

19. Ceballos AGC, Carvalho FM, Araújo TM, Reis EJFB. Avaliação perceptivo-auditiva e fatores associados à alteração vocal em professores. Rev Bras Epidemiol 2011; 14:285-95.

20. Souza CL, Carvalho FM, Araújo TM, Reis EJFB, Lima VMC, Porto LA. Fatores associados a patologias de pregas vocais em professores. Rev Saúde Pública $2011 ; 45: 914-21$.

21. Smith E, Gray SD, Dove H, Kirchner L, Heras H. Frequency and effects of teachers' voice problems. J Voice 1997; 11:81-7.

22. Fuess VL, Lorenz MC. Disfonia em professores do ensino municipal: prevalência e fatores de risco. Rev Bras Otorrinolaringol 2003; 69:807-12. 
23. Provenzano L, Sampaio TMM. Prevalência de disfonia em professores do ensino público estadual afastados de sala de aula. Rev CEFAC 2010; 12: 97-108.

24. Munier C, Kinsella R. The prevalence and impact of voice problems in primary school teachers. Occup Med 2008; 58:74-6.

25. Angelillo M, Di Maio G, Costa G, Angelillo N, Barillari U. Prevalence of occupational voice disorders in teachers. J Prev Med Hyg 2009; 50:26-32.

26. Tuomi K, Oja G. Work ability index. 2nd Ed. Helsinki: Finnish Institute of Occupational Health; 1998.

27. Lapo FR, Bueno BO. Professores, desencanto com a profissão e abandono do magistério. Cad Pesqui 2003; 118:65-88.

28. Jardim R, Barreto SM, Assunção AA. Condições de trabalho, qualidade de vida e disfonia entre docentes. Cad Saúde Pública 2007; 23:2439-61.

29. Simberg S, Sala E, Vehmas K, Laine A. Changes in the prevalence of vocal symptoms among teachers during a twelve-year period. J Voice 2005; 19 : 95-102.

\begin{abstract}
This study aimed to estimate the prevalence of self-reported vocal problems among primary schoolteachers and to identify associated occupational factors, using a cross-sectional design and face-to-face interviews with 967 teachers in 20 public schools in Londrina, Paraná State, Brazil. Prevalence of self-reported vocal problems was $25.7 \%$. Adjusted analyses showed associations with characteristics of the employment relationship (workweek $\geq 40$ hours and poor perception of salaries and health benefits), characteristics of the work environment (number of students per class and exposure to chalk dust and microorganisms), psychological factors (low job satisfaction, limited opportunities to express opinions, worse relationship with superiors, and poor balance between professional and personal life), and violence (insults and bullying). Vocal disorders affected one in four primary schoolteachers and were associated with various characteristics of the teaching profession (both structural and workrelated).
\end{abstract}

Faculty; Voice Disorders; Primary and Secondary Education; Occupational Health
30. Guidini RF, Bertoncello F, Zanchetta S, Dragone MLS. Correlações entre ruído ambiental em sala de aula e voz do professor. Rev Soc Bras Fonoaudiol 2012; 17:398-404.

31. Zwart B, Frings Dresen M, Van Duivenbooden J. Test-retest reliability of the Work Ability Index questionnaire. Occup Med 2002; 52:177-81.

32. Geneid A, Rönkkö M, Airaksinen L, Voutilainen R, Toskala E, Alku P, et al. Pilot study on acute voice and throat symptoms related to exposure to organic dust: preliminary findings from a provocation test. Logoped Phoniatr Vocol 2009; 34:67-72.

33. Vedovato TG, Monteiro MI. Perfil sociodemográfico e condições de saúde e trabalho dos professores de nove escolas estaduais paulistas. Rev Esc Enferm USP 2008; 42:291-7.

34. Ferreira LP, Latorre MRDO, Giannini SPP. A violência na escola e os distúrbios de voz de professores. Distúrb Comun 2011; 23:165-72.

\section{Resumen}

El objetivo de este estudio fue estimar la prevalencia de percepción de problemas vocales frecuentes en maestros de la enseñanza básica e investigar factores ocupacionales asociados. Se trata de un estudio transversal con entrevistas individuales a 967 maestros de 20 escuelas públicas de Londrina, Paraná, Brasil. La percepción de problemas vocales frecuentes fue del 25,7\%. Análisis ajustados revelaron una asociación entre esos problemas y características de las condiciones de trabajo ( $\geq 40$ horas semanales, mala percepción de beneficios salariales y de salud), características del entorno de trabajo (número de alumnos por aula, exposición a polvo de tiza y microorganismos), aspectos psicológicos (menos satisfacción en el trabajo, baja posibilidad de expresar opiniones, peor relación con superiores y equilibrio entre la vida profesional y personal) y violencia (insultos y acoso psicológico). La percepción de los trastornos vocales afecta a uno de cada cuatro maestros de la enseñanza básica y se asocia con varias características de la actividad docente, estructurales y relacionadas con el proceso de trabajo.

Docentes; Trastornos de la Voz; Educación Primaria y Secundaria; Salud Laboral
Recebido em 20/Fev/2015

Versão final reapresentada em 10/Jun/2015

Aprovado em 19/Jun/2015 